\title{
1 A new probe for tracking the presence of E141i food colorant
}

2 Antonio Pérez-Gálvez, José Julian Ríos, and María Roca*

3 Department of Food Phytochemistry, Instituto de la Grasa (CSIC). Avenida Padre García

4 Tejero4, 41012, Sevilla, Spain.

$5 \quad P:+34954691054$

$6 \quad F:+34954691262$

$7 \quad$ * To whom correspondence should be addressed (mroca@ig.csic.es) 


\section{ABSTRACT}

9 HPLC/APCI-MS was applied to the characterization of copper pyropheophytin $a$, the main 10 chlorophyllic derivative present in E-141i, a marketed copper chlorophyll mixture used legally for 11 addition of green hues in food products. Exceptionally, its use is banned in Europe and America 12 for fats and oils and consequently, the characterization of copper pyropheophytin a by $13 \mathrm{HPLC} / \mathrm{MS}$ is critical for olive oil control adulteration and also essential for the detection of this 14 colorant in colored foodstuffs. The $\mathrm{MS}^{2}$ of copper pyropheophytin a shows four fragment ions 15 arising from the loss of phytadiene, a product ion corresponding to the loss of the complete propionic unit at $\mathrm{C} 17$, and product ions corresponding to the loss of phytol and additional methyl

17 groups at $D$ ring. This last fragmentation reaction means the description by first time of the opening of the $D$ ring, showing a new fragmentation pathway where to fit product ions of other chlorophyll derivatives not explained up to now and being an ideal probe for tracking the use of colorant E141i in foods. 


\section{Introduction}

Olive oil production is aimed to meet chemical and organoleptic standards established by the International Olive Council (IOC, 2008), and very different consumers' preferences worldwide. Independently of the classification reached (category) by each olive oil stock, the use of colorants to reinforce color of the olive oil is not allowed in Europe and America and this rule is extended to oils or fats from animal or vegetal origin (EU, 1994; CFR, 2012). E-141i is an authorized food colorant, widely used through the food industry that once added to the oil (or food) causes a re-greening on it, or increases its green hue. This practice is performed to reinforce the consumers' idea that relates green colour in oils with 'virgin' category, to provide oils with intense green appearance to particular markets where it is so demanded, or just to set the colour as copper chlorophylls are very stable. The pigment profile of olive oil has been detailed previously (Gandul-Rojas, Roca-L.Cepero, \& Mínguez-Mosquera, 2000). Pheophytin a and $b$ dominate the profile, reaching almost $90 \%$ of the chlorophyllic pattern, with some traces of native chlorophylls and particular chlorophyll derivatives oxidized on C13. Occasionally, deesterified chlorophyll derivatives have been detected in olive oils from varieties with a high chlorophyllase enzyme activity (eg. Arbequina) and depending on the storage conditions (time and temperature) pyropheophytin a could be present (Gallardo-Guerrero, Gandul-Rojas, Roca, \& Mínguez-Mosquera, 2005). Any other chlorophyll derivative detected in olive oil would indicate an adulteration, and this is the case of chlorophyll derivatives existing in E-141i, which are a mixture of copper derivatives, mainly copper pyropheophytin $a$ as it has been described in a previous work (Roca, Gallardo-Guerrero, Mínguez-Mosquera, \& Gandul-Rojas, 2010). Thus, copper pyropheophytin $a$ is the target compound that should be sought in analyses aimed to determine the presence of E-141i for coloring purposes in olive oil (or in any other food where the use is banned). This compound is a phytylated derivative of chlorophyll, which shows the loss of the methoxycarbonyl group of ring E, and copper (II) inserted in the porphyrin ring (Figure 1). The structural rearrangement of copper pyropheophytin a modifies the spectroscopic characteristics of the native chlorophyll, with batochromic and hypsochromic shifts. Additionally, a modification of the chromatographic properties is also produced. All these features allow the preliminary identification of the copper pyropheophytin a (Roca, Gallardo-Guerrero, MínguezMosquera, \& Gandul-Rojas, 2010, Scotter, Castle, \& Roberts, 2005, Inoue, Furuya, Watanabe, 
Tanaka, Shirai, \& Miyoshi, 1988). Characterization of zinc-containing derivatives by mass spectrometry has been achieved with the use of positive ion fast atom bombardment and tandem mass spectrometry, in order to obtain molecular weight and structural information of the analyzed compounds (van Breemen, \& Canjura, 1991).

The aim of this work was to perform the analysis by mass spectrometry both of the copper pyropheophytin a standard prepared at laboratory from the parent chlorophyll and the one present in E-141i pigment mixtures supplied by different dealers. This work provides new mass spectrometric data that will be useful as reference in the identification of this compound in olive oil samples (or in whatever E141i colored foodstuffs).

\section{Materials and methods}

2.1. Chemicals. Ammonium acetate was supplied by Fluka (Zwijndrecht, The Netherlands), HPLC reagent grade solvents were purchased from Teknokroma (Barcelona, Spain), and analytical grade solvents were supplied by Panreac (Barcelona, Spain). For the preparation, isolation, and purification of chlorophyll derivatives, analytical grade reagents were used (Panreac, Barcelona, Spain). The deionized water used was obtained from a Milli-Q 50 system (Millipore Corp., Milford, MA). Chlorophyll a was purchased from Sigma- Aldrich Co. (Madrid, Spain).

2.2. Samples: The Mg-free derivative (pheophytin) was obtained from chlorophyll a solution in diethyl ether by acidification with 2-3 drops of $5 \mathrm{M} \mathrm{HCl}$ (Sievers, \& Hynninen, 1977). Pyropheophytin a was obtained from pheophytin by reflux-heating at $100 \stackrel{\circ}{ } \mathrm{C}$ in trimethyl pyridine (Schwartz, Woo \& von Elbe, 1981). For the copper complex (Jones, White, Gibbs, \& Denard, 1968) the pigment was dissolved in acetone for the dechelation reaction with an excess of copper (II) ions as chloride and with ascorbic acid to minimize oxidative changes. The samples of colorant E141i were supplied by four different dealers engaged in the production and marketing of food colorants. For analysis, different aliquots were dissolved in acetone.

\subsection{Liquid chromatography/Atmospheric pressure chemical ionization Time-of-Flight} Mass Spectrometry. The liquid chromatograph in the HPLC/APCI-TOF-MS system was a Dionex Ultimate3000RS U-HPLC (Thermo Fisher Scientific, Waltham, MA, USA). A stainless 
steel column $\left(20 \times 0.46 \mathrm{~cm}\right.$ i.d.) packed with $3 \mu \mathrm{m} \mathrm{C}_{18}$ Mediterranea Sea (Teknokroma, Barcelona, Spain) was used. The precolumn $(1 \times 0.4 \mathrm{~cm}$ i.d.) was packed with the same material. Chromatographic separation was performed as earlier described (Roca, Gallardo-Guerrero, Mínguez-Mosquera, \& Gandul-Rojas, 2010) but with modifications as follows. Separation was performed using an elution gradient with the mobile phases $(A)$ water/ion reagent/methanol $(1 / 1 / 8, v / v / v)$ and $(B)$ methanol/acetone $(1 / 1, v / v)$. The ion reagent was $1 \mathrm{M}$ ammonium acetate in water. The column was stored in methanol/water $(1 / 1, \mathrm{v} / \mathrm{v})$. Flow rate was kept constant at $1.25 \mathrm{~mL} / \mathrm{min}$ and the gradient program starts with $75 \% \mathrm{~A}$, then changes to $25 \% \mathrm{~A}$ in $8 \mathrm{~min}$, isocratic $2 \mathrm{~min}$, change to $10 \% \mathrm{~A}$ in $8 \mathrm{~min}$, then to $100 \% \mathrm{~B}$ in $5 \mathrm{~min}$, isocratic $15 \mathrm{~min}$, and return to initial conditions in $5 \mathrm{~min}$. Sequential detection was performed with a photodiode array detector at $430 \mathrm{~nm}$, although the complete spectrrum from $350-800 \mathrm{~nm}$ was recorded. Mass spectrometry was performed using a micrOTOF-QII High Resolution Time-of-Flight mass spectrometer (UHR-TOF) with qQ-TOF geometry (Bruker Daltonics, Bremen, Germany) equipped with an APCI interface. The instrument was operated in positive ion mode using a scan range from $\mathrm{m} / \mathrm{z}$ 50-1200. Mass spectra were acquired in MS fullscan mode and MS-MS spectra were acquired in Auto-MS/MS mode (data-dependent acquisition) and were used for obtaining structural information of compound(s) detected. Collision energy was estimated dynamically based on appropriate values for the mass and stepped across $a+/-10 \%$ magnitude range to ensure good quality fragmentation spectra. The instrument control was performed using Bruker Daltonics HyStar 3.2. Structure of product ions from the parent compound was predicted with the use of Mass Frontier ${ }^{\mathrm{TM}}$ 4.0. This is a software package for the management, evaluation and interpretation of mass spectra, including the automated generation of possible product ions and rearrangement mechanisms, starting from a user-supplied chemical structure. With this feature of the software we can check consistency between a chemical structure and its mass spectrum and recognize the structural differences between spectra of closely related compounds. The program generates a fragmentation scheme for the drawn molecular structure using fragmentation rules of mass spectrometry known in the literature, as well as the selected ionization mode and the number of fragmentation steps (Hufsky, Scheubert, \& Böcker, 2014).

\section{Results and discussion}


111 In our work we applied APCI-MS in positive mode, a soft ionization technique that avoids matrix 112 interferences in FAB-MS, and with enhanced sensitivity for analysis of trace amounts in 113 samples (Harris, Carter, Head, \& Harris, 1995; Aparicio-Ruiz, Riedl \& Schwartz, 2011). Figure 1142 2a shows the HPLC analysis of the standard copper pyropheophytin a complex following the 115 chromatographic and spectroscopic characteristics described before (Roca, Gallardo-Guerrero, 116 Mínguez-Mosquera, \& Gandul-Rojas, 2010): retention time and the maximum wavelengths at 117424 and $654 \mathrm{~nm}$. The four E141i commercial samples analyzed presented similar HPLC traces being copper pyropheophytin a the main chlorophyll derivative quantitatively present in the pigment profile (Roca, Gallardo-Guerrero, Mínguez-Mosquera, \& Gandul-Rojas, 2010), being Figure $2 \mathrm{~b}$ an example. HPLC-APCI-MS analysis showed an extracted ion chromatogram for a theoretical value of $m / z=874.4817 \mathrm{Da}[\mathrm{M}+\mathrm{H}]^{+}$in the standard sample corresponding to copperpyropheophytin $a$, and the corresponding for the E141i sample (Suppl. Fig. 1a and 1b). The maximum of the signal was observed at $32 \mathrm{~min}$ that fits with the elution time of copper pyropheophytin a (Roca, Gallardo-Guerrero, Mínguez-Mosquera, \& Gandul-Rojas, 2010). The accurate mass measured for the protonated molecule at that elution time was $874.4898 \mathrm{Da}$ that means an error of $8.2 \mathrm{mDa}$. The complexity of the pigment mixture in the E141i food colorant could be high, as it may contain up to 15 different chlorophyll derivatives as well as carotenoids (Roca, Gallardo-Guerrero, Mínguez-Mosquera, \& Gandul-Rojas, 2010, Figure 2b), and consequently the possibility of identification by exact mass facilitate (Suppl. Fig. 1) and guarantee the control of adulteration. $\mathrm{MS}^{2}$ allows the isolation of an individual ion for fragmentation and in APCl ion current is associated with one species, so ion abundance is high and the signal of product ions is improved. Table 1 shows the mass spectral product ions in the mass spectra of copper pyropheophytin a standard and in the E141i food colorant. The product ions obtained by $\mathrm{MS}^{2}$ are the same in the standard and in the four E141i samples analysed. The base peak $(m / z=596.1798 \mathrm{Da}, \mathrm{m} / z=596.1805 \mathrm{Da}$ for standard and E141i respectively) corresponds to $\left[\mathrm{M}+\mathrm{H}-\mathrm{C}_{20} \mathrm{H}_{38}\right]^{+}$that is the loss of a phytadiene molecule (fragmentation I in Figure 1), a product ion already described for the zinc-containing chlorophyll derivatives (van Breemen, Canjura, \& Schwartz 1991), which is a favourable process in $\mathrm{MS}^{2}$ of chlorophylls, occurring from the protonated molecule with back transfer of a proton to form phytadiene (Keely, \& Maxwell, 1990), via the McLafferty rearrangement. The second product ion $(\mathrm{m} / \mathrm{z}=$ 
494.1454 Da, $m / z=494.1456 \mathrm{Da}$ for standard and E141i respectively), with $50 \%$ of abundance relative to the base peak, corresponds to the loss of $380 \mathrm{Da}\left(\mathrm{C}_{24} \mathrm{H}_{44} \mathrm{O}_{3}\right)$, which means the loss of the phytyl chain, the complete propionic structure, part of $D$ ring and the keto group at $E$ ring (fragmentation II in Figure 1). With the help of SmartFormula3D ${ }^{\mathrm{TM}}$ module, the consistency of this product ion was checked (exact mass, elemental composition and isotopic pattern). This is the first time that the opening of the ring $D$ is proposed as a possible fragmentation pathway in a chlorophyll derivative compound. Although higher losses $(411,470 \mathrm{Da})$ during $\mathrm{MS}^{\mathrm{n}}$ fragmentation have been found for several chlorophyll compounds (Kostiainen, Hyvärinen, \& Hynninen, 1995; Hyvärinen, \& Hynninen, 1999; Jie, Walker, \& Keely, 2002), any possible explanation for those product ions was detailed so far. We propose a new framework where possibly some of the already described fragmentations may fit. While in most of the chlorophyll derivatives, fragmentation pathway is addressed to processes besides the phytadiene lost, at the $\mathrm{C}$ and $\mathrm{E}$ rings, and loss of the complete $\mathrm{C} 17$ function is not observed (Grese, Cerny, \& Gross, 1989; Jie, Walker, \& Keely, 2002). The complete loss of the propionic structure and even the opening of ring $\mathrm{D}$ observed in pyropheophytins seem to be linked to the scarce functionalization of $\mathrm{C}$ and $\mathrm{E}$ rings that in pyropheophytins only contain the keto function at $\mathrm{C} 13^{1}$. Other product ions at $m / z=550.1793 \mathrm{Da}, \mathrm{m} / \mathrm{z}=550.1808$ for standard and E141i respectively, and $m / z=522.1446 \mathrm{Da} m / z=522.1514$ for standard and E141i respectively $(15 \%$ and $10 \%$ of abundance relative to base peak) correspond to $\left[\mathrm{M}+\mathrm{H}-\mathrm{C}_{21} \mathrm{H}_{39} \mathrm{O}_{2}\right]^{+}$and $\left[\mathrm{M}+\mathrm{H}-\mathrm{C}_{23} \mathrm{H}_{43} \mathrm{O}_{2}\right]^{+}$, respectively. These product ions represent the loss of the phytyl chain plus $\mathrm{COOH}$ (fragmentation III in figure 1) and two additional methyl groups of the propionic group at D ring of the molecule (fragmentation IV in Figure 1). The possibility of the hydrogen back transfer to the macrocycle after the C-17 methylpropionate ester moiety has already proposed for alkyl sulfur chlorophyll derivatives (Pickering, \& Keely, 2007). All the structures arising from the fragmentation pathway of copper pyropheophytin a were simulated with the software Mass Frontier and their consistency was checked with the software module SmartFormula3D ${ }^{\mathrm{TM}}$ that performs an analysis of the experimental mass error and isotopic pattern of the product ions. Occasionally, in some $\mathrm{MS}^{2}$ analysis of the copper pyropheophytin a the base peak is 595.1736 Da that corresponds to the radical cation with the loss of phytyl as a radical species. During description of chlorophyll a allomers by APCI in positive mode (Jie, Walker, \& Keely, 2002) it 
171 was observed a lower abundance ion that corresponds to loss of phytyl as a radical without 172 back transfer of a proton. Generation of radical cations is not a usual process in APCI spectra 173 but their appearance could be explained by the resonance stabilization of the radical species provided by the aromatic ring, which is occupied by copper. This hypothesis has also been claimed using positive ion FAB-MS, where the formation of $\mathrm{M}^{+\bullet}$ is favoured in chlorophylls analysis (Kostiainen, Hyvärinen, \& Hynninen, 1995; van Breemen, Canjura, \& Schwartz 1991) since they contain a delocalized $\pi$-system and their first oxidation potential is low.

\section{Conclusions}

High performance liquid chromatography in combination with atmospheric pressure chemical ionization mass spectrometry provides an excellent advantage in that a selected ion can be traced for study of its fragmentation pattern. Once that pattern has been established, this technique enhances the possibilities of detection of that specific compound that could be present in very low concentrations. $\mathrm{MS}^{2}$ of copper pyropheophytin $a$ is characterized by losses of phytadiene or phytyl, additional losses of the propionic acid at different levels and a new and unexpected rupture of ring D. Up to now, most of the product ions after $M S^{n}$ analysis described in chlorophyll derivatives correspond to the rupture of phytyl and functions around ring E, mainly in $\mathrm{C}_{13^{2}}$ and in $\mathrm{C} 15$. But probably due to the specific configuration of ring $\mathrm{E}$ in copper pyropheophytin $a$, it makes very stable this structure, the rupture around ring $D$ is favoured. This specific and unique fragmentation, gives an ideal probe for tracking the use of colorant E141i in foods, and especially for the forbidden utilization in olive oils.

\section{Acknowledgements}

This work was supported by the Comisión Interministerial de Ciencia y Tecnología (CICYT-EU, Spanish and European Government, AGL 2012-39714) and by Junta de Andalucía (AGR 62712011). We thank Juan Elías Garrido for technical assistance.

\section{References}

Aparicio-Ruiz, R., Riedl, K. M., \& Schwartz, S. J. (2011). Identification and quantification of metallo-chlorophyll complexes in bright green table olives by high-performance liquid 
chromatography-mass spectrometry quadrupole/time-of-flight. Journal of the Agricultural and Food Chemistry, 59, 11100-11108.

Code of Federal Regulations (2012) Title 21, Chapter I, Subchapter A, Part 73, Subpart A.

European Union. (1994). Official Journal of the Commission of the European Communities. Regulation No. 94/36/CE, L237.

Gallardo-Guerrero, L., Gandul-Rojas, B., Roca, M., \& Mínguez-Mosquera, M.I. (2005). Effect of storage on the original pigment profile of spanish virgin olive oil. Journal of the American Oil Chemistry Society, 82, 33-39.

Gandul-Rojas, B., Roca-L.Cepero, M., \& Mínguez-Mosquera, M.I. (2000). Use of chlorophyll and carotenoid pigment composition to determine authenticity of virgin olive oil. Journal of the American Oil Chemistry Society, 77, 853-858.

Grese, R.P., Cerny, R.L., \& Gross, M.L. (1989). Determination of structure and properties of modified chlorophylls by using fast atom bombardment combined with tandem mass spectrometry. Journal of the American Society for Mass Spectrometry, 1, 72-84.

Harris, P.G., Carter, J.F., Head, R.N., Harris, R.P., Eglinton G., \& Maxwell, J.R. (1995). Identification of chlorophyll transformation products in zooplankton faecal pellets and marine sediment extracts by liquid chromatography/mass spectrometry atmospheric pressure chemical ionization. Rapid Communications in Mass Spectrometry, 9, 1177-1183.

Hufsky, F., Scheubert, K., \& Böcker, S. (2014). Computational mass spectrometry for smallmolecule fragmentation. Trends in Analytical Chemistry, 53, 41-48.

Hyvärinen, K., \& Hynninen, P.H. (1999). Liquid chromatographic separation and mass spectrometric identification of chlorophyll $b$ allomers. Journal of Chromatography A, 837, 107116.

Inoue, H., Furuya, K., Watanabe, K., Tanaka, K., Shirai, T., \& Miyoshi, E. (1988). Separation and determination of copper (II) chlorophylls by reversed-phase high performance liquid chromatography. Analytical sciences, 4, 599-603. 

phase liquid chromatography mass spectrometry and tandem mass spectrometry of chlorophyll a allomers. Rapid Communications in Mass Spectrometry, 16, 473-479. Jones, I.D., White, R.C., Gibbs, E., \& Denard, C.D. (1968). Absortion spectra of copper and zinc complexes of pheophytins and pheophorbides. Journal of the Agricultural and Food Chemistry $16,80-83$.

Keely, B.J., \& Maxwell J.R. (1990). Fast atom bombardment mass spectrometric and tandem mass spectrometric studies of some functionalized tetrapyrroles derived from chlorophylls $a$ and b. Energy \& Fuels, 4, 737-741. of the $13^{2}$-epimers of $13^{2}$-hydroxychlorophyll a. Rapid Communications in Mass Spectrometry, 9, 555-558.

Pickering, M.D., \& Keely, B. J. (2007). Alkyl sulfur chlorophyll derivatives: preparation and liquid chromatography-multistage tandem mass spectrometric characterization of analogues of naturally occurring sedimentary species. Organic Geochemistry, 39, 1046-1050.

Roca, M., Gallardo-Guerrero, L., Mínguez-Mosquera, M. I., \& Gandul-Rojas, B. (2010). Control of olive oil adulteration with copper-chlorophyll derivatives. Journal of the Agricultural and Food Chemistry, 58, 51-56.

Schwartz, S. J., Woo, S. L., \& von Elbe, J. H. (1981). High-performance liquid chromatography of chlorophylls and their derivatives in fresh and processed spinach. Journal of the Agricultural and Food Chemistry, 29, 533-535.

Scotter, M. J., Castle, L., \& Roberts, D. (2005). Method development and HPLC analysis of retail foods and beverages for copper chlorophyll (E141[i]) and chlorophyllin (E141[ii]) food colouring materials. Food Additives \& Contaminants, 22, 1163-1175. derivatives on cellulose layers. Journal of Chromatography A, 134, 359-364. 
251 van Breemen, R. B., Canjura, F. L., \& Schwartz, S. J. (1991). Identification of chlorophyll 252 derivatives by mass spectrometry. Journal of the Agricultural and Food Chemistry, 39, 14522531456.

254 
256 Figure 1.- Structures of chlorophyll a, copper pyropheophytin $a$ and the main fragment ions 257 detected by $\mathrm{APCl}-\mathrm{MS}^{2}$ after copper pyropheophytin a fragmentation. I: $\mathrm{m} / z=596.1849 \mathrm{Da}$; II: $258 m / z=494.1454 \mathrm{Da} ;$ III: $m / z=550.1793 \mathrm{Da} ;$ IV: $m / z=522.1446 \mathrm{Da}$ (theoretical mass values).

259 Figure 2.- HPLC trace (at $430 \mathrm{~nm}$ ) corresponding to the analysis of the standard copper 260 pyropheophytin $a(A)$ and to one of the marketed E141i food colorant (B). Insert is the UV261 visible spectrum of copper pyropheophytin a complex. 1, Copper pyropheophytin a, c, 262 carotenoids and $\mathrm{p}$, pheophorbide and pheophytin derivatives identified previously (Roca, 263 Gallardo-Guerrero, Mínguez-Mosquera, \& Gandul-Rojas, 2010). 
Chlorophyll a Copper pyropheophytin a
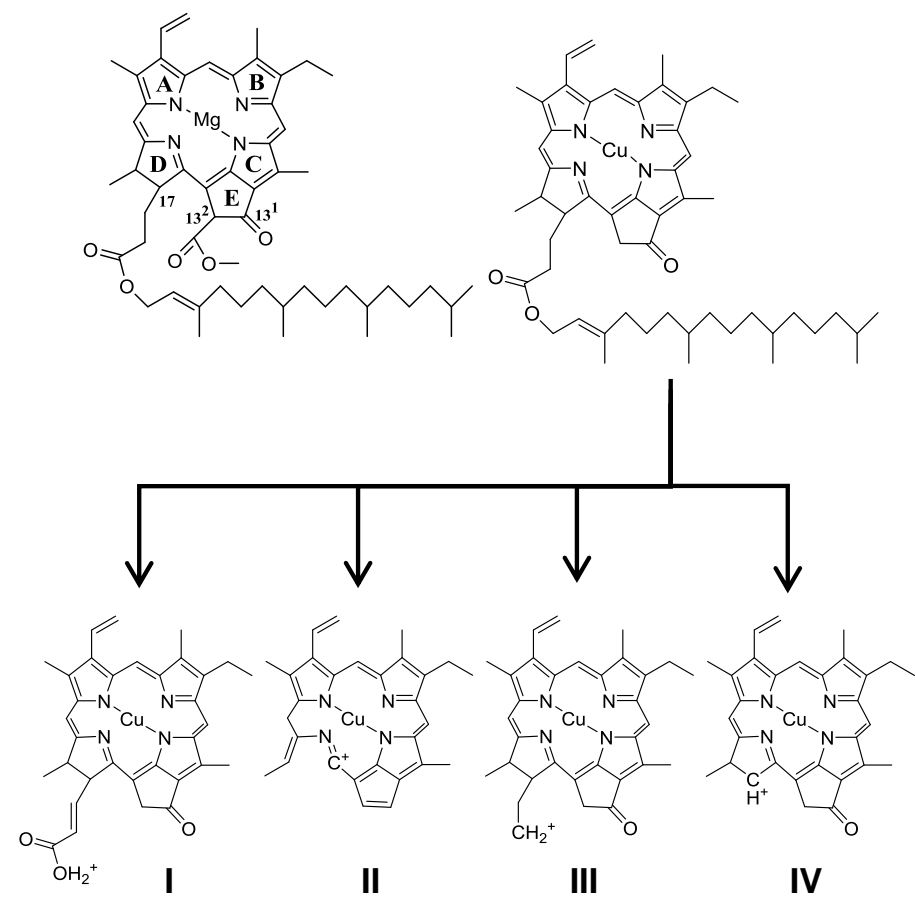


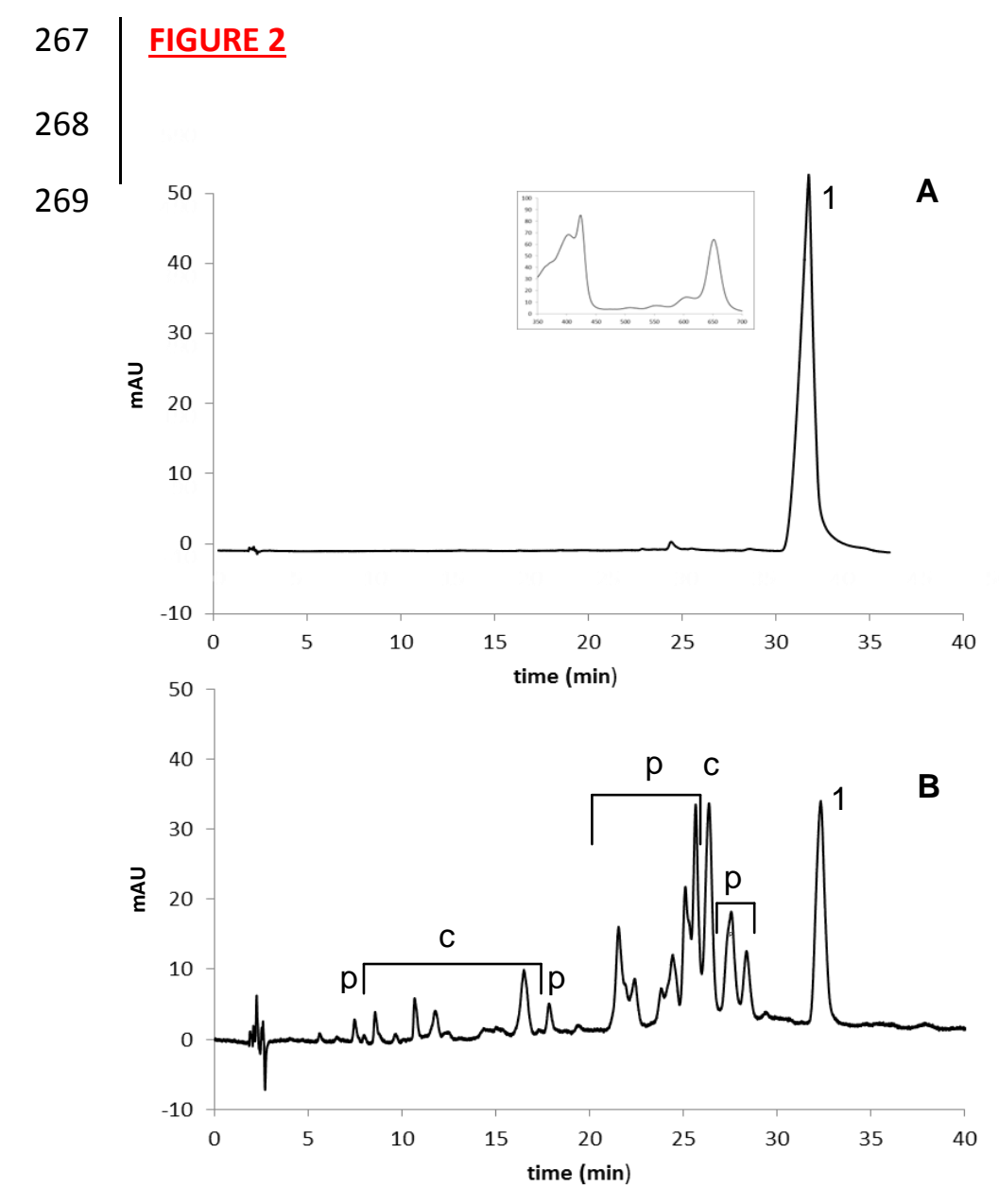

